\title{
Prediction of ground subsidence in Samcheok City, Korea using artificial neural networks and GIS
}

\author{
Ki-Dong Kim · Saro Lee $\cdot$ Hyun-Joo Oh
}

Published online: 7 February 2009

(C) Springer-Verlag 2009

\section{Erratum to: Environ Geol}

\section{DOI 10.1007/s00254-008-1492-9}

1. [Page 1, Affiliation] Author, Ki-Dong Kim, have two affiliations as follows:

- Ecosystem Survey Team, Ecosystem Assessment Division, National Institute of Environmental Research (NIER), Environmental Research Complex, Kyungseodong, Seo-gu, Incheon, 404-708, Korea

- Department of Geoinformation Engineering, Sejong University, 98 Gunja-dong, Gwangjin-gu, Seoul 143-747, South Korea

The online version of the original article can be found under doi:10.1007/s00254-008-1492-9.

\section{K.-D. Kim}

Ecosystem Survey Team, Ecosystem Assessment Division, National Institute of Environmental Research (NIER),

Environmental Research Complex, Kyungseo-dong,

Seo-gu, Incheon 404-708, Korea

\section{K.-D. Kim}

Department of Geoinformation Engineering, Sejong University,

98 Gunja-dong, Gwangjin-gu, Seoul 143-747, South Korea

S. Lee $(\square)$

Geoscience Information Center, Korea Institute of Geoscience and Mineral Resources (KIGAM), 92 Gwahang-no,

Yuseong-gu, Daejeon 305-350, South Korea

e-mail: leesaro@kigam.re.kr

H.-J. Oh

Department of Earth System Sciences, Yonsei University,

262 Seongsanno, Seodaemun-gu, Seoul 120-749, South Korea
2. [Page 8, Table 3]

In online publication, the orders of factors and weights mismatched in Table 3.

Then, we have correctly matched the orders of factors and weights as follows: 
Table 3 Weights of each factor estimated by neural networks considered in this study

\begin{tabular}{|c|c|c|c|c|c|c|c|c|c|c|c|c|c|}
\hline \multirow[t]{2}{*}{ Factors } & \multicolumn{13}{|l|}{ Run } \\
\hline & 1 & 2 & 3 & 4 & 5 & 6 & 7 & 8 & 9 & 10 & Mean & S.D. & N.W.* \\
\hline Geology & 0.1631 & 0.1414 & 0.1513 & 0.1337 & 0.1434 & 0.1135 & 0.1285 & 0.1627 & 0.1516 & 0.1326 & 0.1422 & 0.0157 & 1.4031 \\
\hline Slope & 0.1174 & 0.0925 & 0.0960 & 0.1266 & 0.0848 & 0.1021 & 0.1124 & 0.0727 & 0.0770 & 0.1318 & 0.1013 & 0.0204 & 1.0000 \\
\hline Land use & 0.1856 & 0.1803 & 0.1667 & 0.1433 & 0.1786 & 0.1652 & 0.1584 & 0.1930 & 0.1890 & 0.1777 & 0.1738 & 0.0153 & 1.7150 \\
\hline Depth of drift & 0.0818 & 0.1236 & 0.0921 & 0.1465 & 0.0942 & 0.1445 & 0.0776 & 0.0937 & 0.0895 & 0.1283 & 0.1072 & 0.0260 & 1.0577 \\
\hline Distance from drift & 0.0998 & 0.1178 & 0.0783 & 0.1311 & 0.0920 & 0.1337 & 0.1293 & 0.0938 & 0.0953 & 0.1055 & 0.1077 & 0.0192 & 1.0625 \\
\hline Depth of ground water & 0.2237 & 0.1833 & 0.2362 & 0.1675 & 0.2486 & 0.1688 & 0.2144 & 0.2524 & 0.2452 & 0.1785 & 0.2119 & 0.0343 & 2.0908 \\
\hline Permeability & 0.1286 & 0.1610 & 0.1793 & 0.1515 & 0.1585 & 0.1722 & 0.1794 & 0.1316 & 0.1525 & 0.1456 & 0.1560 & 0.0179 & 1.5397 \\
\hline
\end{tabular}

3. [Page 6, Fig. 4]

The legend of Fig. 4(c) Land use has errors. We have corrected that.

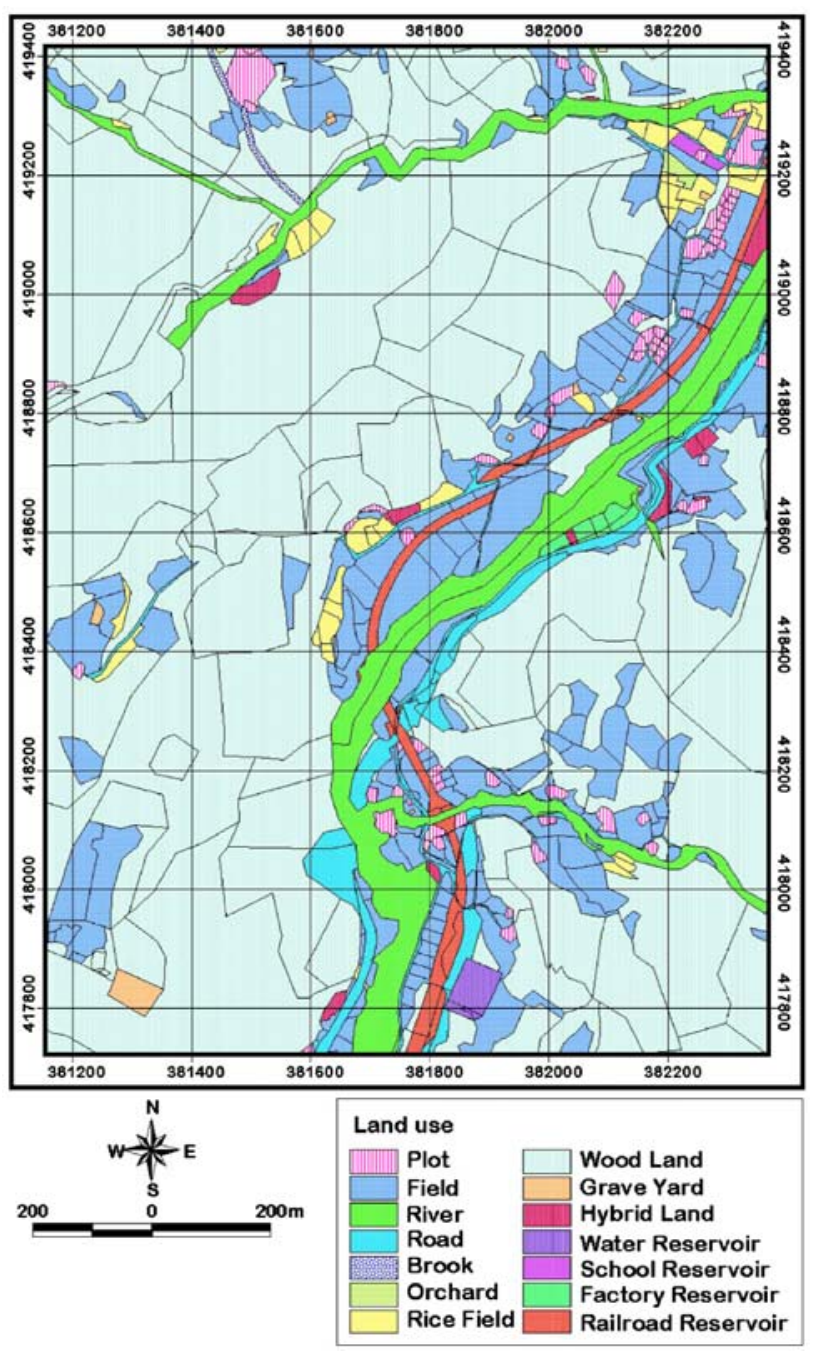

Fig. 4 Input factors 